\title{
Hadamard upper bound on optimum joint decoding capacity of Wyner Gaussian cellular MAC
}

\author{
Muhammad Zeeshan Shakir ${ }^{1} 2^{*}$, Tariq S Durrani ${ }^{2}$ and Mohamed-Slim Alouini ${ }^{1}$
}

\begin{abstract}
This article presents an original analytical expression for an upper bound on the optimum joint decoding capacity of Wyner circular Gaussian cellular multiple access channel (C-GCMAC) for uniformly distributed mobile terminals (MTs). This upper bound is referred to as Hadamard upper bound (HUB) and is a novel application of the Hadamard inequality established by exploiting the Hadamard operation between the channel fading matrix $\mathbf{G}$ and the channel path gain matrix $\boldsymbol{\Omega}$. This article demonstrates that the actual capacity converges to the theoretical upper bound under the constraints like low signal-to-noise ratios and limiting channel path gain among the MTs and the respective base station of interest. In order to determine the usefulness of the HUB, the behavior of the theoretical upper bound is critically observed specially when the inter-cell and the intra-cell time sharing schemes are employed. In this context, we derive an analytical form of HUB by employing an approximation approach based on the estimation of probability density function of trace of Hadamard product of two matrices, i.e., $\mathbf{G}$ and $\boldsymbol{\Omega}$. A closed form of expression has been derived to capture the effect of the MT distribution on the optimum joint decoding capacity of C-GCMAC. This article demonstrates that the analytical HUB based on the proposed approximation approach converges to the theoretical upper bound results in the medium to high signal to noise ratio regime and shows a reasonably tighter bound on optimum joint decoding capacity of Wyner GCMAC.
\end{abstract}

\section{Introduction}

The ever growing demand for communication services has necessitated the development of wireless systems with high bandwidth and power efficiency $[1,2]$. In the last decade, recent milestones in the information theory of wireless communication systems with multiple antenna and multiple users have offered additional newfound hope to meet this demand [3-11]. Multiple input multiple output (MIMO) technology provides substantial gains over single antenna communication systems, however the cost of deploying multiple antennas at the mobile terminals (MTs) in a cellular network can be prohibitive, at least in the immediate future $[3,8]$. In this context, distributed MIMO approach is a means of realizing the gains of MIMO with single antenna terminals in a cellular network allowing a gradual migration to a

\footnotetext{
* Correspondence: muhammad.shakir@kaust.edu.sa

'Division of Physical Sciences and Engineering, King Abdullah University of Science and Technology, KAUST, Thuwa1 23599-6900, Makkah Province, Kingdom of Saudi Arabia

Full list of author information is available at the end of the article
}

true MIMO cellular network. This approach requires some level of cooperation among the network terminals which can be accomplished through suitably designed protocols [4-6,12-16]. Toward this end, in the last few decades, numerous articles have been written to analyze various cellular models using information theoretic argument to gain insight into the implications on the performance of the system parameters. For an extensive survey on this literature, the reader is referred to $[5,6,17-19]$ and the references there in.

The analytical framework of this article is inspired by analytically tractable model for multicell processing (MCP) as proposed in [7], where Wyner incorporated the fundamental aspects of cellular network into the framework of the well known Gaussian multiple access channel (MAC) to form a Gaussian cellular MAC (GCMAC). The majority of the MCP models preserve fundamental assumptions which has initially appeared in Wyner's model, namely (i) interference is considered only from two adjacent cells; (ii) path loss variations among the MTs and the respective base stations (BSs) 
are ignored; (iii) the interference level at a given BS from neighboring users in adjacent cells is characterized by a deterministic parameter $0 \leq \Omega \leq 1$, i.e., the collocation of MTs (users). ${ }^{a}$

\section{A. Background and related study}

In [7], Wyner considered optimal joint processing of all BSs by exploiting cooperation among the BSs. It has been shown that intra-cell time division multiple access (TDMA) scheme is optimal and achieves capacity. Later, Shamai and Wyner considered a similar model with frequency flat fading scenario and more conventional decoding schemes, e.g., single-cell processing (SCP) and two-cell-site processing schemes $[5,6]$. It has also been shown that the optimum joint decoding strategy is distinctly advantageous over intra-cell TDMA scheme and fading between the terminals in a communication link increases the capacity with the increase in the number of jointly decoded users. Later, in [20] Wyner model has been modified by employing multiple transmitting and receiving antennas at both ends of the communication link in the cellular network where each BS is also composed of multiple antennas. Recently, new results have been published by further modifying the Wyner model with shadowing [21].

Recently, Wyner model has been investigated to account for randomly distributed users, i.e., non-collocated users [21-24]. In [22], an instant signal-interference-ratio (SIR) and averaged throughout for randomly distributed users have been derived by employing TDMA and code division multiple access (CDMA) schemes. It has been shown that the Wyner model is accurate only for the system with sufficient number of simultaneous users. It has also been shown that for MCP scenario, the CDMA outperforms the inter-cell TDMA which is opposite to the original results of Wyner, where inter-cell TDMA is shown to be capacity achieving [7]. Later in the article, similar kind of analysis has also been presented for downlink case which is out of scope of this article. The readers are referred to [22] and references there in.

Although the Wyner model is mathematically tractable, but still it is unrealistic with respect to practical cellular systems that the users are collocated with the BSs and offering deterministic level of interference intensity to the respective BS. As a consequence, another effort has been made to derive an analytical capacity expression based on random matrix theory $[21,23]$. Despite the fact that the variable-user density is used in this article, the analysis is only valid under the asymptotic assumptions of large number of MTs $K$, i.e., $K \rightarrow \infty$ and infinite configuration of number of cooperating BSs $N$, i.e., $N \rightarrow \infty$ such that
$\frac{K}{N} \rightarrow c \in(0,1)[17,21,23,24]$. On the contrary, the main contribution of our article is to offer non-asymptotic approach to derive information theoretic bound on Wyner GCMAC model where finite number of BSs arranged in a circle are cooperating to jointly decode the user's data.

\section{B. Contributions}

In this article, we consider a circular version of Wyner GCMAC (by wrap around the linear Wyner model to form a circle) which we refer to as circular GCMAC (CGCMAC) throughout the article [12]. We consider an architecture where the BSs can cooperate to jointly decode all user's data, i.e., macro-diversity. Thus, we dispense with cellular structure altogether and consider the entire network of the cooperating BSs and the users as a network-MIMO system [12]. Each user has a link to each BS and BSs cooperate to jointly decode all user's data. The summary of main contributions of this article are described as follows. We derive a non-asymptotic analytical upper bound on the optimum joint decoding capacity of Wyner C-GCMAC by exploiting the Hadamard inequality for finite cellular network-MIMO setup. The bound is referred to as Hadamard upper bound (HUB). In this study, we alleviate the Wyner's original assumption by assuming that the MTs are uniformly distributed across the cells in Wyner C-GCMAC.

In first part of this article, we introduce the derivation of Hadamard inequality and its application to derive information theoretic bound on optimum joint decoding capacity which we referred to as theoretical HUB. The theoretical results of this article are exploited further to study the effect of variable path gains offered by each user in adjacent cells to the BS of interest (due to variable-user density). The performance analysis of first part of this article includes the presentation of capacity expressions over multi-user and single-user decoding strategies with and without intra-cell and inter-cell TDMA schemes to determine the existence of the proposed upper bound. In the second part of this article, we derive the analytical form of HUB by approximating the probability density function (PDF) of Hadamard product of channel fading matrix $\mathbf{G}$ and channel path gain matrix $\Omega$. The closed form representation of HUB is presented in the form of Meijer's G-Function. The performance and comparison description of analytical approach includes the presentation of information theoretic bound over the range of signal-to-noise ratios (SNRs) and the calculation of mean area spectral efficiency (ASE) over the range of cell radii for the system under consideration.

This article is organized as follows. In Section II, system model for Wyner C-GCMAC is recast in Hadamard 
matrix framework. Next in Section III, the Hadamard inequality is derived as Theorem 3.3 based on Theorem 3.1 and Corollary 3.2. While in Section IV, a novel application of the Hadamard inequality is employed to derive the theoretical upper bound on optimum joint decoding capacity. This is followed by the several simulation results for a single-user and the multi-user scenarios that validate the analysis and illustrate the effect of various time sharing schemes on the performance of the optimum joint decoding capacity for the system under consideration. In Section V, we derive a novel analytical expression for an upper bound on optimum joint decoding capacity. This is followed by numerical examples and discussions in Section VI that validate the theoretical and analytical results, and illustrate the accuracy of the proposed approach for realistic cellular network-MIMO systems. Conclusions are presented in Section VII.

Notation: Throughout the article, $\mathbb{R}^{N \times 1}$ and $\mathbb{C}^{N \times 1}$ denote $N$ dimensional real and complex vector spaces, respectively. Furthermore, $\mathbb{P}^{N \times 1}$ denotes $N$ dimensional permutation vector spaces which has 1 at some specific position in each column. Moreover, the matrices are represented by an uppercase boldface letters, as an example, the $N \times M$ matrix $\mathrm{A}$ with $N$ rows and $M$ columns are represented as $\mathbf{A}^{N \times M}$. Similarly, the vectors are represented by a lowercase boldface italic version of the original matrix, as an example, a $N \times 1$ column vector $\boldsymbol{a}$ is represented as $\boldsymbol{a}^{N \times 1}$. An element of the matrix or a vector is represented by the non-boldface letter representing the respective vector structure with subscripted row and column indices, as an example $a_{n, m}$ refers to the element referenced by row $n$ and column $m$ of a matrix $\mathrm{A}^{N \times M}$. Similarly, $a_{k}$ refers to element $k$ of the vector $\boldsymbol{a}^{N \times 1}$. Scalar variables are always represented by a non-boldface italic characters. The following standard matrix function are defined as follows: $(\cdot)^{T}$ denotes the non-Hermitian transpose; $(\cdot)^{H}$ denotes the Hermitian transpose; $\operatorname{tr}($.$) denotes the trace of a square$ matrix; det (.) and $|\cdot|$ denote the determinant of a square matrix; $\|\mathbf{A}\|$ denotes the norm of the matrix $\mathbf{A}$; $\mathbb{E}[\cdot]$ denotes the expectation operator and ( $\left.{ }^{\circ}\right)$ denotes the Hadamard operation (element wise multiplication) between the two matrices.

\section{Wyner Gaussian cellular Mac model}

\section{A. System model}

We consider a circular version of Gaussian cellular MAC (C-GCMAC), where $N=6$ cells are arranged in a circle such that the BSs are located in the center of each cell as shown in Figure 1[12,25]. The inspiration of small number of cooperating BSs is based on [26] where we have shown the existence of circular cellular structure found in city centers of large cities in the UK, i.e.,

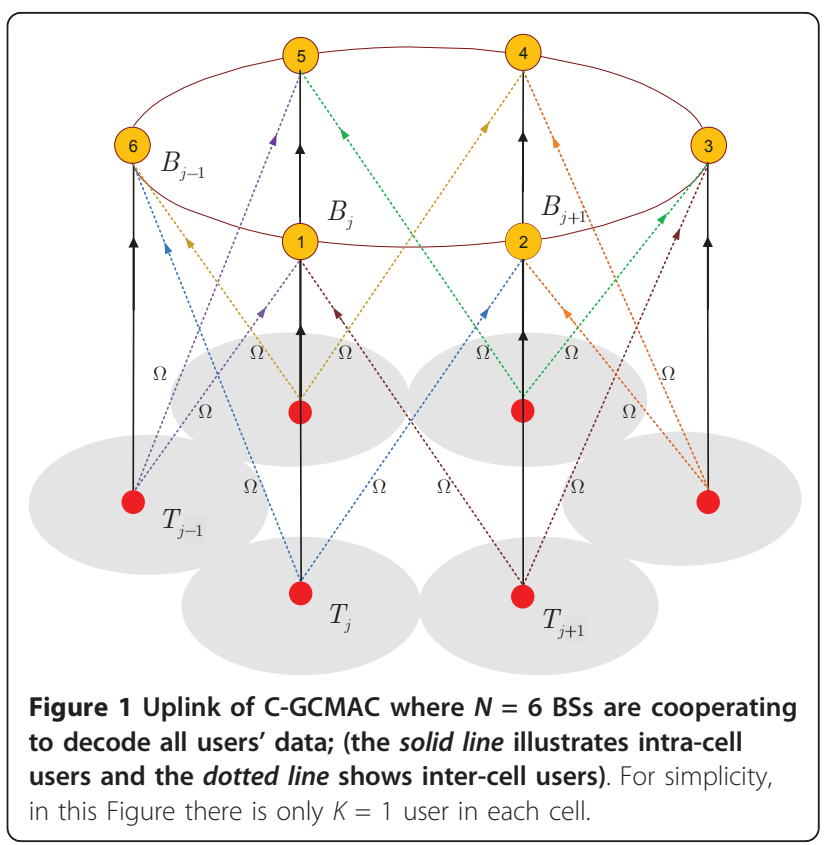

Glasgow, Edinburgh, and London. It has been shown that BSs can cooperate to jointly decode all users data. Furthermore, we employed a circular array instead of the typical linear array because of its analytical tractability. In the limiting scenario of the large number of cooperating BSs, these two array topologies are expected to be equivalent [25]. Moreover, each cell has $K$ MTs such that there are $M=N K$ MTs (users) in the entire system. Assuming a perfect symbol and frame synchronism at a given time instant, the received signal at each of the BS is given by[12] $]^{\mathrm{b}}$

$$
y_{j}=\sum_{l=1}^{K} h_{B_{j} T_{j}}^{l} x_{j}^{l}+\sum_{i= \pm 1} \sum_{l=1}^{K} h_{B_{j} T_{j+i}}^{l} x_{j+i}^{l}+z_{j}
$$

where $\left\{B_{j}\right\}_{j=1}^{N}$ are the BSs; $\left\{T_{j}\right\}_{j=1}^{N}$ are the source MTs, $K$ for each cell; $x_{j}^{l}$ represents the symbol transmitted by the $l$ th $\mathrm{MT} T_{j}$ in $j$ th cell. Furthermore, the MTs are assumed to transmit independent, zero mean complex symbols such that each subject to an individual average power constraint, i.e., $\mathbb{E}\left[\left\|x_{j}^{l}\right\|^{2}\right] \leq P$ for all $(j, l)=(1, \ldots$, $N) \times(1, \ldots, K)$ and $z_{j}$ is an independent and identically distributed (i.i.d) complex circularly symmetric (c.c.s) Gaussian random variable with variance $\sigma_{z}^{2}$ such that each $z_{j} \sim \mathcal{C N}\left(0, \sigma_{z}^{2}\right)$. Finally, $h_{B_{j} T_{j}}^{l}$ is identified as the resultant channel fading component between the $l$ th MT $T_{j}$ and the BS $B_{j}$ in $j$ th cell. Similarly, $h_{B_{j} T_{j+i}}^{l}$ is the resultant channel fading component between the $l$ th MT $T_{j+i}$ in $(j+i)$ th cell for $i= \pm 1$, belonging to adjacent cells and BS $B_{j}$ in $j$ th cell. In general, we refer $h_{B_{j} T_{j}}^{l}$ and 
as the intra-cell and inter-cell resultant channel fading components, respectively, and may be expressed as

$$
h_{B_{j} T_{j+i}}^{l}=\left(g_{B_{j} T_{j+i}}^{l} \circ \Omega_{B_{j} T_{j+i}}^{l}\right) \text { for }\{i=0, \pm 1\},
$$

where $\left({ }^{\circ}\right)$ denotes the Hadamard product between the two gains; the fading gain $g_{B_{j} T_{j+i}}^{l}$ is the small scale fading coefficients which are assumed to be ergodic c.c.s Gaussian processes (Rayleigh fading) such that each $g_{B_{j} T_{j+i}}^{l} \sim C N(0,1)$ and $\Omega_{B_{j} T_{j+i}}$ denotes frequency flat-path gain that strictly depends on the distribution of the MTs such that each $\Omega_{B_{j} T_{j+i}} \sim \mathcal{U}(0,1)$ (path gains between the users and respective BSs follow normalized Uniform distribution). In particular, the path loss between the MTs and the BSs can be calculated according to the normalized path loss mode1[20]

$$
\Omega_{B_{j} T_{j+i}}^{l}=\left(\frac{d_{B_{j} T_{j}}^{l}}{d_{B_{j} T_{j+i}}^{l}}\right)^{\eta / 2} \text { for }\{i=0, \pm 1\},
$$

where $d_{B_{i} T_{j}}^{l}$ and $d_{B_{j} T_{i+i}}^{l}$ are the distances along the line of sight of the transmission path between the intra-cell and inter-cell MTs to the respective BS of the interest, respectively, such that $d_{B_{j} T_{j}}^{l} \leq d_{B_{j} T_{j+i}}^{l}$ for $(l=1 \ldots K)$. Furthermore, the path gains between the inter-cell MTs and the respective BS are normalized with respect to the distances between the intra-cell MTs and respective BS such that $0 \leq \Omega_{B_{j} T_{j+i}}^{l} \leq 1$ in $(j+i)$ th cell for $\{i=0, \pm 1\}$ [20]. Also, the $\eta$ is the path loss exponent and we assumed it is 4 for urban cellular environment [2]. It is to note that these two components of the resultant composite fading channel are mutually independent as they are because of different propagation effects. Therefore, the C-GCMAC model in (1) can be transformed into the framework of Hadamard product as follows:

$$
y_{j}=\sum_{l=1}^{K}\left(g_{B_{j} T_{j}}^{l} \circ \Omega_{B_{j} T_{j}}^{l}\right) x_{j}^{l}+\sum_{i= \pm 1} \sum_{l=1}^{K}\left(g_{B_{j} T_{j+i}}^{l} \circ \Omega_{B_{j} T_{j+i}}^{l}\right) x_{j+i}^{l}+z_{j} .
$$

For notation convenience, the entire signal model over C-GCMAC can be more compactly expressed as a vector memoryless channel of the form

$$
y=\mathbf{H} x+z,
$$

where $y \in \mathbb{C}^{N \times 1}$ is the received signal vector, $\boldsymbol{x} \in$ $\mathbb{C}^{N K \times 1}$ represents the transmitted symbol vector by all the MTs in the system, $z \in \mathbb{C}^{N \times 1}$ represents the noise vector of i.i. d c.c.s Gaussian noise samples with $\mathbb{E}[z]=0, \mathbb{E}\left[z z^{H}\right]=\sigma_{z}^{2} \mathbf{I}_{N}$ and $\mathbf{H} \in \mathbb{C}^{N \times N K}$ is the resultant composite channel fading matrix. The matrix $\mathbf{H}$ is defined as the Hadamard product of the channel fading and channel path gain matrices given by ${ }^{\mathrm{c}}$

$$
\mathbf{H}_{N, K} \triangleq\left(\mathbf{G}_{N, K} \circ \boldsymbol{\Omega}_{N, K}\right),
$$

where $\mathbf{G}_{N, K} \in \mathbb{C}^{N \times N K}$ such that $\mathbf{G}_{N, K} \sim \mathcal{C N}\left(\mathbf{0}, \mathbf{I}_{N}\right)$ and $\boldsymbol{\Omega}_{N, K} \in \mathbb{R}^{N \times N K}$ such that $\boldsymbol{\Omega}_{N, K} \sim \mathcal{U}(0,1)$. The modeling of channel path gain matrix $\boldsymbol{\Omega}_{N, K}$ for a single-user and the multi-user environments can be well understood from the following Lemma.

Lemma 2.1: (Modeling of Channel Path Gain Matrix)

Let $\mathbf{S}$ be a circular permutation operator, viewed as $N \times$ $N$ matrix relative to the standard basis for $\mathbb{R}^{N}$. For a given circular cellular setup where initially we assumed $K=1$ and $N=6$ such that there are $M=N K=6$ users in the system. Let $\left\{e_{1}, e_{2}, \ldots, e_{6}\right\}$ be the standard row basis vectors for $\mathbb{R}^{N}$ such that $e_{i}=\mathbf{S} e_{i+1}$ for $i=1,2, \ldots, N$. Therefore, the circular shift operator matrix $\mathbf{S}$ relative to the defined row basis vectors, can be expressed as $[27,28]$

$$
\mathbf{S}=\left(\begin{array}{llllll}
0 & 1 & 0 & 0 & 0 & 0 \\
0 & 0 & 1 & 0 & 0 & 0 \\
0 & 0 & 0 & 1 & 0 & 0 \\
0 & 0 & 0 & 0 & 1 & 0 \\
0 & 0 & 0 & 0 & 0 & 1 \\
1 & 0 & 0 & 0 & 0 & 0
\end{array}\right)
$$

The matrix $\mathbf{S}$ is real and orthogonal, hence $\mathbf{S}^{-1}=\mathbf{S}^{T}$ and also the basis vectors are orthogonal for $\mathbb{R}^{N}$.

- Symmetrical channel path gain matrix: In this scenario, the structure of the channel path gain matrix is typically circular for a single-user case. Therefore, the path gains between the MTs $T_{j+i}$ for $\{i=0, \pm 1\}$ and the respective BSs $B_{j}$ are deterministic and can be viewed as a row vector of the resultant $N \times N$ circular channel path gain matrix $\boldsymbol{\Omega}$. Mathematically, the first row of the channel matrix may be expressed as ${ }^{\mathrm{d}}$ $\boldsymbol{\Omega}(1,:)=\left(\Omega_{B_{j} T_{j}}, \Omega_{B_{j} T_{j+i}} 0,0,0, \Omega_{B_{j} T_{j-i}}\right)$, where $\Omega_{B_{j} T_{j}}$ is the path gain between the intra-cell MTs $T_{j}$ and the respective BSs in $j$ th cell and $\Omega_{B_{j} T_{j+i}}$ for $i= \pm 1$ is the channel path gain between the MTs $T_{j+i}$ for $i= \pm 1$ in the adjacent cells and the respective BSs in $j$ th cell. In this context, it is known that the circular matrix $\boldsymbol{\Omega}$ can be expressed as a linear combination of powers of the shift operator $\mathbf{S}[27,28]$. Therefore, the resultant circular channel path gain matrix (symmetrical) for $K=1$ active user in each cell can be expressed as

$$
\boldsymbol{\Omega}_{N, 1}=\mathbf{I}_{N}+\Omega_{B_{j} T_{j+1}} \mathbf{S}+\Omega_{B_{j} T_{j-1}} \mathbf{S}^{T},
$$

where $I_{N}$ is $N \times N$ identity matrix; $\mathrm{S}$ is the shift operator and $\Omega_{B_{j} T_{j \pm 1}} \sim \mathcal{U}(0,1)$. Furthermore, for the multiuser scenario the channel path gain matrix becomes block-circular matrix such that (8) may be extended as

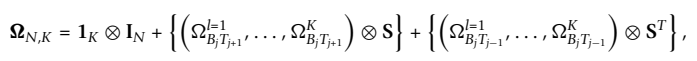


where $\mathbf{1}_{K}$ denotes $1 \times K$ all ones vector and $(\otimes)$ denotes the Kronecker product.

- Unsymmetrical channel path gain matrix: In this scenario, the MTs (users) in the adjacent cells are randomly distributed across the cells in the entire system. Therefore, the channel path gain matrix is not deterministic, and hence, the resultant matrix is no more circular. In this setup, the channel path gain matrix for single-user scenario can be mathematically modeled as follows:

$$
\boldsymbol{\Omega}_{N, 1}=\mathbf{I}_{N}+\hat{\mathbf{\Omega}}_{N, 1} \circ \mathbf{S}+\hat{\boldsymbol{\Omega}}_{N, 1} \circ \mathbf{S}^{T},
$$

where $\hat{\boldsymbol{\Omega}}_{N, 1} \sim \mathcal{U}(0,1)$.

Similarly, for the multi-user scenario the channel path gain matrix in (10) may be extended as follows:

$$
\boldsymbol{\Omega}_{N, K}=\mathbf{1}_{K} \otimes \mathbf{I}_{N}+\hat{\mathbf{\Omega}}_{N, K} \circ\left\{\mathbf{1}_{K} \otimes \mathbf{S}\right\}+\hat{\boldsymbol{\Omega}}_{N, K} \circ\left\{\mathbf{1}_{K} \otimes \mathbf{S}^{T}\right\} .
$$

\section{B. Definitions}

Now, we describe the following definitions which we used frequently throughout the article in discussions and analysis.

i. Intra-cell TDMA: a time sharing scheme where only one user in each cell in the system is allowed to transmit simultaneously at any time instant.

ii. Inter-cell TDMA: a time sharing scheme where only one cell in the system is active at any time instant such that each local user inside the cell is allowed to transmit simultaneously. The users in other cells in the system are inactive at that time instant.

iii. Channel path gain $(\Omega)$ : normalized distance dependent path loss offered by intra-cell and intercell MTs to the BS of interest.

iv. MCP: a transmission strategy, where a joint receiver decodes all users data jointly (uplink); while the BSs can transmit information for all users in the system (downlink).

v. SCP: a transmission strategy where the BSs can only decode the data from their local users, i.e., intra-cell users and consider the inter-cell interference from the inter-cell users as a Gaussian noise (uplink); while the BSs can transmit information only for their local users, i.e., intra-cell users (downlink).

\section{Information theory and Hadamard inequality}

In this section, a novel expression for an upper bound on optimum joint decoding capacity based on Hadamard inequality is derived [12]. The upper bound is referred to as HUB. Let us assume that the receiver has perfect channel state information (CSI) while the transmitter knows neither the statistics nor the instantaneous CSI. In this case, a sensible choice for the transmitter is to split the total amount of power equally among all data streams and consequently, an equal power transmission scheme takes place [4-6,12]. The justification for adopting this scheme, though not optimal, originates from the so-called maxmin property which demonstrates the robustness of the above mentioned technique for maximizing the capacity of the worst fading channel [3-6]. Under these circumstances, the most commonly used figure of merit in the analysis of MIMO systems is the normalized total sum-rate constraint, which in this article is referred to as the optimum joint decoding capacity. Following the argument in [8], one can easily show that optimum joint decoding capacity of the system of interest is

$$
\begin{aligned}
& \mathrm{C}_{\text {opt }}(p(\mathbf{H}), \gamma)=\frac{1}{N} \mathcal{I}(x ; y \mid \mathbf{H}), \\
& =\frac{1}{N} \mathbb{E}\left[\log _{2} \operatorname{det}\left(\mathbf{I}_{N}+\gamma \mathbf{H H}^{H}\right)\right],
\end{aligned}
$$

where $p(\mathbf{H})$ signifies that the fading channel is ergodic with density $p(\mathbf{H}) ; \mathbf{I}_{N}$ is a $N \times N$ identity matrix and $\gamma$ is the SNR. Here, the BSs are assumed to be able to jointly decode the received signals in order to detect the transmitted vector $\boldsymbol{x}$. Applying the Hadamard decomposition (6), the Hadamard form of (13) may be expressed as

$$
\mathrm{C}_{\mathrm{opt}}(p(\mathbf{H}), \gamma)=\frac{1}{N} \mathbb{E}\left[\log _{2} \operatorname{det}\left(\mathbf{I}_{N}+\gamma(\mathbf{G} \circ \boldsymbol{\Omega})(\mathbf{G} \circ \boldsymbol{\Omega})^{H}\right] .\right.
$$

Theorem 3.1: (Hadamard Product)

Let $\mathbf{G}$ and $\boldsymbol{\Omega}$ be an arbitrary $N \times M$ matrices. Then, we have [29-31]

$$
\mathbf{G} \circ \boldsymbol{\Omega}=\mathbf{P}_{N}^{T}(\mathbf{G} \otimes \boldsymbol{\Omega}) \mathbf{P}_{M},
$$

where $\mathbf{P}_{N}$ and $\mathcal{P}_{M}$ are $N^{2} \times N$ and $M^{2} \times M$ partial permutation matrices, respectively (in some of the literatures these matrices are referred to as selection matrices [29]). The jth column of $\mathbf{P}_{N}$ and $\mathbf{P}_{M}$ has 1 in its $((j-1)$ $N+j)$ th and $((j-1) M+j)$ th positions, respectively, and zero elsewhere.

Proof: See [[31], Theorem 2.5].

In particular if $N=M$, then we have

$$
\mathbf{G} \circ \boldsymbol{\Omega}=\mathbf{P}_{N}^{T}(\mathbf{G} \otimes \boldsymbol{\Omega}) \mathbf{P}_{N} .
$$

\section{Corollary 3.2: (Hadamard Product)}

This corollary lists several useful properties of the partial permutation matrices $\mathbf{P}_{N}$ and $\mathbf{P}_{M}$. For brevity, the partial permutation matrices $\mathbf{P}_{N}$ and $\mathbf{P}_{M}$ will be denoted by $\mathbf{P}$ unless it is necessary to emphasize the order. In 
the same way, the partial permutation matrices $\mathbf{Q}_{N}$ and $\mathbf{Q}_{M}$, defined below, are denoted by Q[12]. . $^{\mathrm{e}}$

i. $\mathbf{P}_{N}$ and $\mathbf{P}_{M}$ are the only matrices of zeros and onces that satisfy (15) for all $\mathbf{G}$ and $\Omega$.

ii. $\mathbf{P}^{T} \mathbf{P}=\mathbf{I}$ and $\mathbf{P} \mathbf{P}^{T}$ is a diagonal matrix of zeros and ones, so $0 \leq \operatorname{diag} 0\left(\mathbf{P P}^{T}\right) \leq 1$.

iii. There exists a $N^{2} \times\left(N^{2}-N\right)$ matrix $\mathbf{Q}_{N}$ and $M^{2}$ $\times\left(M^{2}-M\right)$ matrix $\mathbf{Q}_{M}$ of zeros and ones such that $\pi \triangleq(\mathbf{P} \mathbf{Q})$ is the permutation matrix. The matrix $\mathbf{Q}$ is not unique but for any choice of $\mathbf{Q}$, following holds:

$\mathbf{P}^{T} \mathbf{Q}=\mathbf{0} ; \quad \mathbf{Q}^{T} \mathbf{Q}=\mathbf{I} ; \quad \mathbf{Q Q}^{T}=\mathbf{I}-\mathbf{P P}^{T}$.

iv. Using the properties of a permutation matrix together with the definition of $\pi$ in (iii); we have

$$
\pi \pi^{T}=\left(\begin{array}{ll}
\mathbf{P} & \mathbf{Q}
\end{array}\right)\left(\begin{array}{l}
\mathbf{P}^{T} \\
\mathbf{Q}^{T}
\end{array}\right)=\mathbf{P P}^{T}+\mathbf{Q Q}^{T}=\mathbf{I} \text {. }
$$

Theorem 3.3: (Hadamard Inequality)

Let $\mathbf{G}$ and $\Omega$ be an arbitrary $N \times M$ matrices. Then $[29,30,32]$

$$
\mathbf{G G}^{H} \circ \Omega \Omega^{H}=(\mathbf{G} \circ \boldsymbol{\Omega})(\mathbf{G} \circ \boldsymbol{\Omega})^{H}+\Gamma_{(\mathbf{P}, \mathbf{Q})},
$$

where $\Gamma_{(\mathbf{P}, \mathbf{Q})}=\mathbf{P}_{N}^{T}(\mathbf{G} \otimes \boldsymbol{\Omega}) \mathbf{Q}_{M} \mathbf{Q}_{M}^{T}(\mathbf{G} \otimes \boldsymbol{\Omega})^{H} \mathbf{P}_{N}$ and we called it the Gamma equality function. From (17), we can obviously deduce [29]

$$
\mathbf{G G}^{H} \circ \boldsymbol{\Omega} \boldsymbol{\Omega}^{H} \geq(\mathbf{G} \circ \boldsymbol{\Omega})(\mathbf{G} \circ \boldsymbol{\Omega})^{H} .
$$

This inequality is referred to as the Hadamard inequality which will be employed to derive the theoretical and analytical HUB on the capacity (14).

Proof: Using the well-known property of the Kronecker product between two matrices $\mathbf{G}$ and $\Omega$, we have [33]

$$
\mathbf{G G}^{H} \otimes \mathbf{\Omega} \boldsymbol{\Omega}^{H}=(\mathbf{G} \otimes \boldsymbol{\Omega})(\mathbf{G} \otimes \boldsymbol{\Omega})^{H}
$$

using Corollary 3.2(iii) i.e., $\left(\mathbf{P}_{M} \mathbf{P}_{M}^{T}+\mathbf{Q}_{M} \mathbf{Q}_{M}^{T}\right)=\mathbf{I}$, subsequently we have

$$
\begin{aligned}
\mathbf{G G}^{H} \otimes \boldsymbol{\Omega} \boldsymbol{\Omega}^{H} & =(\mathbf{G} \otimes \boldsymbol{\Omega})\left(\mathbf{P}_{M} \mathbf{P}_{M}^{T}+\mathbf{Q}_{M} \mathbf{Q}_{M}^{T}\right)(\mathbf{G} \otimes \boldsymbol{\Omega})^{H}, \\
& =(\mathbf{G} \otimes \boldsymbol{\Omega}) \mathbf{P}_{M} \mathbf{P}_{M}^{T}(\mathbf{G} \otimes \boldsymbol{\Omega})^{H}+(\mathbf{G} \otimes \boldsymbol{\Omega}) \mathbf{Q}_{M} \mathbf{Q}_{M}^{T}(\mathbf{G} \otimes \boldsymbol{\Omega})^{H},
\end{aligned}
$$

multiply each term by partial permutation matrix $\mathbf{P}$ of appropriate order to ensure Theorem 3.1, we have

$$
\begin{aligned}
\mathbf{P}_{N}^{T}\left(\mathbf{G G}^{H} \otimes \boldsymbol{\Omega} \boldsymbol{\Omega}^{H}\right) \mathbf{P}_{N}= & \mathbf{P}_{N}^{T}(\mathbf{G} \otimes \boldsymbol{\Omega}) \mathbf{P}_{M} \mathbf{P}_{M}^{T}(\mathbf{G} \otimes \boldsymbol{\Omega})^{H} \mathbf{P}_{N} \\
& +\mathbf{P}_{N}^{T}(\mathbf{G} \otimes \boldsymbol{\Omega}) \mathbf{Q}_{M} \mathbf{Q}_{M}^{T}(\mathbf{G} \otimes \boldsymbol{\Omega})^{H} \mathbf{P}_{N}
\end{aligned}
$$

subsequently, we can prove that

$$
\mathbf{G G}^{H} \circ \boldsymbol{\Omega} \boldsymbol{\Omega}^{H}=(\mathbf{G} \circ \boldsymbol{\Omega})(\mathbf{G} \circ \boldsymbol{\Omega})^{H}+\Gamma_{(\mathbf{P}, \mathbf{Q})}
$$

and

$$
\mathbf{G G}^{H} \circ \boldsymbol{\Omega} \boldsymbol{\Omega}^{H} \geq(\mathbf{G} \circ \boldsymbol{\Omega})(\mathbf{G} \circ \boldsymbol{\Omega})^{H} .
$$

This completes the proof of Theorem 3.3.

An alternate proof of (18) is provided as Appendix A.

\section{Theoretical Hub}

In this section, we first introduce the theoretical upper bound by employing the Hadamard inequality (18). Later, we demonstrate the behavior of the theoretic upper bound when various time sharing schemes are employed. It is to note that the aim of employing the time sharing schemes is to illustrate the usefulness of HUB in practical cellular network. The upper bound on optimum joint decoding capacity using the Hadamard inequality (Theorem 3.3 ) is derived as

$$
\begin{aligned}
& \mathrm{C}_{\text {opt }}(p(\mathbf{H}), \gamma) \leq \overline{\mathrm{C}}_{\text {opt }}(p(\mathbf{H}), \gamma) \\
& =\frac{1}{N} \mathbb{E}\left[\log _{2} \operatorname{det}\left(\mathbf{I}_{N}+\gamma\left(\mathbf{G G}^{H}\right) \circ\left(\boldsymbol{\Omega} \boldsymbol{\Omega}^{H}\right)\right)\right] .
\end{aligned}
$$

Now, in the following sub-sections we analyze the validity of the HUB on optimum joint decoding capacity w.r.t a single-user and the multi-user environments under limiting constraints.

\section{A. Single-user environment i. Low inter-cell interference regime}

For a single-user case, as the inter-cell interference intensity among the MTs and the respective BSs is negligible, i.e., $\Omega \rightarrow 0$, the actual optimum joint decoding capacity approaches to the theoretical HUB on the capacity, since $\mathbf{G}$ and $\boldsymbol{\Omega}$ becomes diagonal matrices and (18) holds equality results such that

$$
\mathbf{G G}^{H} \circ \boldsymbol{\Omega} \boldsymbol{\Omega}^{H}=(\mathbf{G} \circ \boldsymbol{\Omega})(\mathbf{G} \circ \boldsymbol{\Omega})^{H} .
$$

It is to note that this is the scenario in cellular network when the MTs in adjacent cells are located far away from the BS of interest. Practically, the MTs in the adjacent cells which are located at the edge away from the BS of interest are offering negligible path gain.

Proof: To arrive at (21), we first notice from (17) that $\mathbf{P}_{N}^{T}(\mathbf{G} \otimes \boldsymbol{\Omega}) \mathbf{Q}_{M} \mathbf{Q}_{M}^{T}=0$ only when $\mathbf{G}$ and $\boldsymbol{\Omega}$ are the diagonal matrices. Using corollary 3.2(iii), i.e., $\mathbf{Q}_{M} \mathbf{Q}_{M}^{T}=\mathbf{I}-\mathbf{P}_{M} \mathbf{P}_{M}^{T}$, we have $\mathbf{P}_{N}^{T}(\mathbf{G} \otimes \boldsymbol{\Omega})\left(\mathbf{I}-\mathbf{P}_{M} \mathbf{P}_{M}^{T}\right)=0$ such that

$$
\mathbf{P}_{N}^{T}(\mathbf{G} \otimes \boldsymbol{\Omega})=\mathbf{P}_{N}^{T}(\mathbf{G} \otimes \boldsymbol{\Omega}) \mathbf{P}_{M} \mathbf{P}_{M^{\prime}}^{T}
$$


multiply both sides by $(\mathbf{G} \otimes \boldsymbol{\Omega})^{H} \mathbf{P}_{N}$, we have

$$
\mathbf{P}_{N}^{T}(\mathbf{G} \otimes \boldsymbol{\Omega})(\mathbf{G} \otimes \boldsymbol{\Omega})^{H} \mathbf{P}_{N}=\mathbf{P}_{N}^{T}(\mathbf{G} \otimes \boldsymbol{\Omega}) \mathbf{P}_{M} \mathbf{P}_{M}^{T}(\mathbf{G} \otimes \boldsymbol{\Omega})^{H} \mathbf{P}_{N}
$$

using the well property of Kronecker product between two matrices $\mathbf{G}$ and $\boldsymbol{\Omega}$ which states that $(\mathbf{G} \otimes \boldsymbol{\Omega})(\mathbf{G} \otimes$ $\boldsymbol{\Omega})^{H}=\mathbf{G G}^{H} \otimes \mathbf{\Omega} \boldsymbol{\Omega}^{H}$, we have

$$
\mathbf{P}_{N}^{T}\left(\mathbf{G G}^{H} \otimes \boldsymbol{\Omega} \boldsymbol{\Omega}^{H}\right) \mathbf{P}_{N}=\mathbf{P}_{N}^{T}(\mathbf{G} \otimes \boldsymbol{\Omega}) \mathbf{P}_{M} \mathbf{P}_{M}^{T}(\mathbf{G} \otimes \boldsymbol{\Omega})^{H} \mathbf{P}_{N},
$$

ensuring Theorem 3.1, we finally arrived at

$$
\mathbf{G G}^{H} \circ \boldsymbol{\Omega} \boldsymbol{\Omega}^{H}=(\mathbf{G} \circ \boldsymbol{\Omega})(\mathbf{G} \circ \boldsymbol{\Omega})^{H} .
$$

This completes the proof of (21).

Therefore, by employing (21) in the low inter-cell interference regime, we have

$$
\begin{aligned}
& \bar{C}_{\text {opt }}(p(\mathbf{H}), \gamma)=\lim _{\Omega \rightarrow 0} \frac{1}{N} \mathbb{E}\left[\log _{2} \operatorname{det}\left(\mathbf{I}_{N}+\gamma\left(\mathbf{G G}^{H}\right) \circ\left(\boldsymbol{\Omega} \boldsymbol{\Omega}^{H}\right)\right)\right] \\
& =C_{\text {opt }}(p(\mathbf{H}), \gamma) .
\end{aligned}
$$

The summary of theoretical HUB on optimum joint decoding capacity over flat faded C-GCMAC for $K=$ 1 is shown in Figure 2. The curves are obtained over 10,000 Monte Carlo simulation trials of the resultant channel fading matrix $\mathbf{H}$. It can be seen that the theoretical bound is relatively lose in the medium to high SNR regime as compared to the bound in the low SNR regime (compare the black solid curve using (14) with the red dashed curve using (20)). The upper bound is the consequence of the fact that the determinant is increasing in the space of semi-definite positive matrices $\mathbf{G}$ and $\boldsymbol{\Omega}$. It can be seen that in the limiting environment, such as when $\Omega \rightarrow 0$, the actual optimum joint decoding capacity approaches the theoretical upper bound (compare the curve with red square markers and the black dashed-dotted curve in Figure 2). It is to note that the channel path gain $\Omega$ among the MTs in the adjacent cells and BS of interest may be negligible when the users are located at the edge away from the BS of interest, i.e., MTs are located far away from the BS of interest such that $\Omega$ $\rightarrow 0$.

\section{ii. Tightness of HUB-low SNR regime}

In this sub-section, we show that the actual optimum joint decoding capacity converges to the theoretical HUB in the low SNR regime whereas in the high SNR regime, the offset from the actual optimum capacity is almost constant [12]. In general, if $\Delta$ is the absolute gain inserted by the theoretical upper bound on $C_{\mathrm{opt}}$ which may be expressed as

$$
\Delta=\overline{\mathrm{C}}_{\text {opt }}(p(\mathbf{H}), \gamma)-\mathrm{C}_{\mathrm{opt}}(p(\mathbf{H}), \gamma),
$$

and asymptotically tends to zero as $\gamma \rightarrow 0$, given as

$$
\Delta_{0}=\lim _{\gamma \rightarrow 0} \gamma \frac{1}{N} \mathbb{E}[\operatorname{tr}(\Gamma(\mathbf{P}, \mathbf{Q}))] .
$$

Proof: Using (24), we have

$$
\begin{aligned}
\Delta & =\frac{1}{N} \mathbb{E}\left[\log _{2}\left(\frac{\operatorname{det}\left(\mathbf{I}_{N}+\gamma\left(\mathbf{G ~ G}^{H} \circ \boldsymbol{\Omega} \boldsymbol{\Omega}^{H}\right)\right)}{\operatorname{det}\left(\mathbf{I}_{N}+\gamma(\mathbf{G} \circ \boldsymbol{\Omega})\left(\mathbf{G} \circ \mathbf{\Omega}^{H}\right)\right)}\right)\right] \\
& =\frac{1}{N} \mathbb{E}\left[\log _{2}\left(\frac{1+\gamma \operatorname{tr}\left(\mathbf{G} \mathbf{G}^{H} \circ \boldsymbol{\Omega} \boldsymbol{\Omega}^{H}\right)+\mathcal{O}_{0}\left(\gamma^{2}\right)}{1+\gamma \operatorname{tr}\left((\mathbf{G} \circ \boldsymbol{\Omega})\left(\mathbf{G} \circ \mathbf{\Omega}^{H}\right)\right)+\mathcal{O}_{1}\left(\gamma^{2}\right)}\right)\right],
\end{aligned}
$$

where we have made a use of property $\operatorname{det}(\mathbf{I}+\gamma \mathbf{A})=1+\gamma \operatorname{tr} \mathbf{A}+\mathcal{O}\left(\gamma^{2}\right)[33],{ }^{f}$ hence using (17), the tightness on the bound becomes

$$
\begin{aligned}
& =\frac{1}{N} \mathbb{E}\left[\log _{2}\left(\frac{1+\gamma \operatorname{tr}\left((\mathbf{G} \circ \boldsymbol{\Omega})\left(\mathbf{G} \circ \mathbf{\Omega}^{H}\right)\right)+\gamma \operatorname{tr}(\Gamma(\mathbf{P}, \mathbf{Q}))}{1+\gamma \operatorname{tr}\left((\mathbf{G} \circ \mathbf{\Omega})\left(\mathbf{G} \circ \mathbf{\Omega}^{H}\right)\right)}\right)\right] \\
& =\frac{1}{N} \mathbb{E}\left[\log _{2}\left(1+\frac{\gamma \operatorname{tr}(\Gamma(\mathbf{P}, \mathbf{Q}))}{1+\gamma \operatorname{tr}\left((\mathbf{G} \circ \boldsymbol{\Omega})\left(\mathbf{G} \circ \mathbf{\Omega}^{H}\right)\right)}\right]\right. \\
& =\frac{1}{N} \mathbb{E}\left[\log _{2}(1+\gamma \operatorname{tr}(\Gamma(\mathbf{P}, \mathbf{Q})))\right],
\end{aligned}
$$

in limiting case, using Taylor series expansion we have

$$
\Delta=\frac{1}{N} \mathbb{E}\left[\gamma \operatorname{tr}\left(\Gamma_{(\mathbf{P}, \mathbf{Q})}\right)-\frac{1}{2} \gamma^{2}\left(\operatorname{tr}\left(\Gamma_{(\mathbf{P}, \mathbf{Q})}\right)\right)^{2}+\frac{1}{3} \gamma^{3}\left(\operatorname{tr}\left(\Gamma_{(\mathbf{P}, \mathbf{Q})}\right)\right)^{3}-\cdots\right],
$$

ignoring the terms with higher order of $\gamma$, the asymptotic gain inserted by HUB on optimum joint decoding capacity becomes

$$
\Delta_{0}=\lim _{\gamma \rightarrow 0} \gamma \frac{1}{N} \mathbb{E}[\operatorname{tr}(\Gamma(\mathbf{P}, \mathbf{Q}))] .
$$

This completes the proof of (25).

It is demonstrated in Figure 2 that as $\gamma \rightarrow 0$, the gain inserted by the upper bound $\Delta=\Delta_{0} \approx 0$ (compare the black solid curve with the red dashed curve). It can be seen from the figure that the theoretical HUB on optimum capacity is loose in the high range of SNR regime and comparably tight in the low SNR regime, and hence $\overline{\mathrm{C}}_{\text {opt }}(p(\mathrm{H}), \gamma) \approx \mathrm{C}_{\text {opt }}(p(\mathbf{H}), \gamma)$.

\section{iii. Inter-cell TDMA scheme}

Note that (21) holds if and only if $\Gamma_{(\mathbf{P}, \mathbf{Q})}=0$, which is mathematically equivalent to $\mathbf{P}_{N}^{T}(\mathbf{G} \otimes \boldsymbol{\Omega}) \mathbf{Q}_{M} \mathbf{Q}_{M}^{T}=0$. It is found that for a single-user case, i.e., $K=1$ by employing inter-cell TDMA, i.e., $\Omega=0$, the matrices $\mathbf{G}_{N, 1}$ and $\boldsymbol{\Omega}_{N, 1}$ become diagonal and $\Gamma_{(\mathbf{P}, \mathbf{Q})}=0$. This is considered as a special case in GCMAC decoding when each BS only decodes its own local users (intra-cell users) and there is no inter-cell interference from the adjacent cells. Hence, the resultant channel fading matrix is a diagonal matrix such that for the given $\mathbf{G}_{N, 1}$ and $\boldsymbol{\Omega}_{N, 1}$ (21) holds and we have 


$$
\mathrm{C}_{\mathrm{opt}}^{\mathrm{TDMA}}(p(\mathrm{H}), \gamma K)=\overline{\mathrm{C}}_{\mathrm{opt}}(p(\mathbf{H}), \gamma)=\mathrm{C}_{\mathrm{opt}}(p(\mathbf{H}), \gamma) \text {. }
$$

The same has been shown in Figure 2. The black dashed-dotted curve and the curve with red square marker illustrate optimum capacity and theoretical HUB, respectively, when inter-cell interference is negligible, $\mathrm{i}$. e., using (23). Next, the curve with green circle marker shows the capacity when inter-cell TDMA is employed, i.e., using (26).

\section{B. Multi-user environment}

In this section, we demonstrate the behavior of the theoretical HUB when two implementation forms of time sharing schemes are employed in multi-user environment. One is referred to as inter-cell TDMA, intra-cell narrowband scheme (TDMA, NB), and other is intercell TDMA, intra-cell wideband scheme [12]. We refer the later scheme as inter-cell time sharing, wideband scheme, (ICTS, WB) throughout the discussions. It is to note that SCP is employed only to determine the application of our bound for realistic cellular network.

\section{i. Inter-cell TDMA, intra-cell narrow-band scheme (TDMA, \\ $N B)$}

In multi-user case, when there are $K$ active users in each cell, then the channel matrix is no longer diagonal, and hence $(21)$ is not valid and $\Gamma_{(\mathbf{P}, \mathbf{O})} \neq 0$. However, the

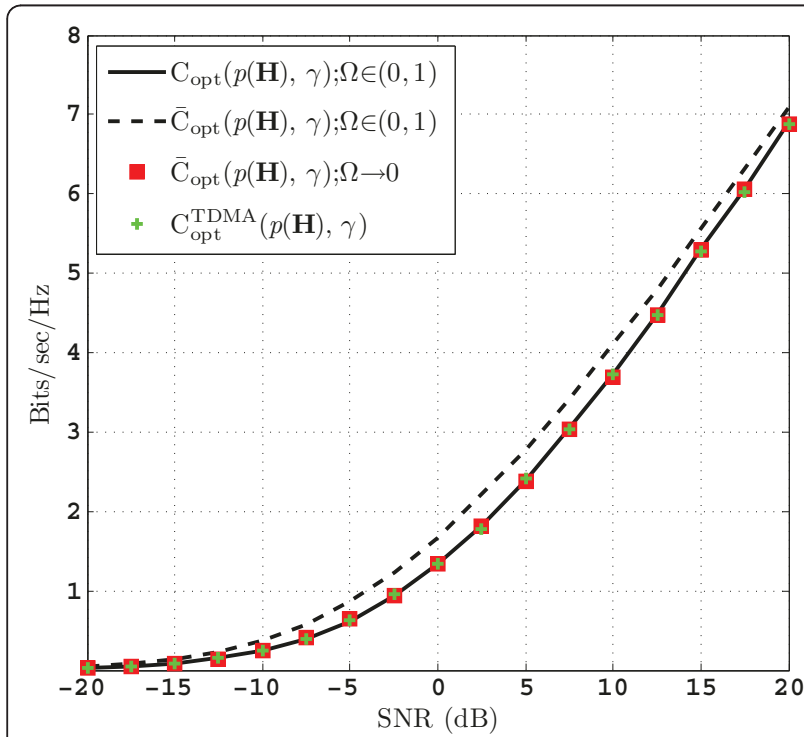

Figure 2 Summary of optimum joint decoding capacity and the Hadamard upper bound on optimum capacity; the black solid curve illustrates the capacity using (14); the red dashed curve illustrates theoretical HUB on capacity using (20); the black dashed-dotted curve and the curve with red square marker illustrate optimum capacity and theoretical HUB, respectively, when inter-cell interference is negligible using (23); the curve with green circle marker shows capacity when inter-cell TDMA is employed using (26) results of single-user case is still valid when intra-cell TDMA scheme is employed in combination with intercell TDMA (TDMA, NB) scheme. If the multi-user resultant channel fading matrix $\mathbf{H}_{N, K}$ is expressed as (6), then by exploiting the TDMA, NB scheme the rectangular resultant channel fading matrix $\mathbf{H}_{N, K}$ may be reduced to $\mathbf{H}_{N, 1}$ and may be expressed as

$$
\mathbf{H}_{N, 1}=\left(\mathbf{G}_{N, 1} \circ \boldsymbol{\Omega}_{N, 1}\right),
$$

where $\mathbf{G}_{N, 1}$ and $\boldsymbol{\Omega}_{N, 1}$ are exactly diagonal matrices as discussed earlier in single-user case. The capacity in this case becomes

$$
\begin{aligned}
& \mathrm{C}_{\mathrm{opt}}^{\mathrm{TDMA}, \mathrm{NB}}(p(\mathbf{H}), \gamma K)=\frac{1}{N} \mathbb{E}\left[\log _{2} \operatorname{det}\left(\mathbf{I}_{N}+\gamma \mathbf{H}_{N, 1} \mathbf{H}_{N, 1}^{H}\right)\right] \\
& =\overline{\mathrm{C}}_{\mathrm{opt}}^{\mathrm{TDMA}, \mathrm{NB}}(p(\mathbf{H}), \gamma K) .
\end{aligned}
$$

The actual optimum capacity offered by this scheduling scheme is equal to its upper bound based on the Hadamard inequality. The scenario is simulated and shown in Figure 3a,b for $K=5$ and 10, respectively. It is to note that the capacity in this figure is normalized with respect to the number of users and the number of cells. It can be seen that the actual optimum capacity and the upper bound on the optimum capacity are identical when TDMA, NB scheme is employed in multiuser environment (compare the curves with red circle markers with the black solid curves in Figure 3a,b).

\section{ii. Inter-cell time sharing, wide-band scheme, (ICTS, WB)}

It is well known that the increase in number of users to be decoded jointly increases the channel capacity $[5,6,13-16]$. Let us consider a scenario in the multi-user environment without intra-cell TDMA, i.e., there are $K$ active users in each cell and they are allowed to transmit simultaneously at any time instant. Mathematically, the local intra-cell users are located along the main diagonal of a rectangular channel matrix $\mathbf{H}_{N, \mathrm{~K}}$. The capacity in this case when only inter-cell TDMA scheme (ICTS, WB) is employed becomes

$$
\begin{aligned}
& \mathrm{C}_{\mathrm{opt}}^{\mathrm{ICTS}, \mathrm{WB}}(p(\mathbf{H}), \gamma)=\frac{1}{N} \mathbb{E}\left[\log _{2} \operatorname{det}\left(\mathbf{I}_{N}+\gamma \mathbf{H}_{N, K} \mathbf{H}_{N, K}^{H}\right)\right] \\
& <\overline{\mathrm{C}}_{\mathrm{opt}}^{\mathrm{ICTS}, \mathrm{WB}}(p(\mathbf{H}), \gamma K) .
\end{aligned}
$$

The capacity by employing ICTS, WB scheme for $K=$ 5 and $K=10$ is shown in Figure 3a,b, respectively. The theoretical upper bound on the capacity using Hadamard inequality by employing ICTS, WB scheme is also shown in this figure (compare the blue solid curve with the red dashed curve). It is observed that the difference between the actual capacity offered by ICTS, WB 
scheme and its theoretical upper bound increases with the increase in number of intra-cell users to be jointly decoded in the multi-user case. An an example, at $\gamma=$ $20 \mathrm{~dB}$ and for $K=5$ the relative difference in capacity due to HUB is $6.5 \%$ and similarly the relative difference is raised to $12 \%$ for $K=10$. Thus, using an inequality (18), multi-user decoding offers $\log _{2}(K)$ times higher non-achievable capacity as compared to actual capacity offered by this scheme. Also, it is well known that the overall performance of ICTS scheduling scheme is superior to the TDMA scheme due to the advantages of wideband transmission (compare the black solid curves with the blue solid curves in Figure 3a,b). The results are summarized in Table 1 to illustrate the existence of HUB for cooperative and non-cooperative BSs in cellular network.

\section{Analytical Hub}

In this section, we approximate the PDF of Hadamard product of channel fading matrix $\mathbf{G}$ and channel path gain matrix $\Omega$ as the PDF of the trace of the Hadamard product of these two matrices, i.e., $\mathbf{G}$ and $\boldsymbol{\Omega}$. Recall from (20) (section 4), an upper bound on optimum joint decoding capacity (14) using the Hadamard inequality (Theorem 3.3) is derived as

$$
\begin{aligned}
& \mathrm{C}_{\text {opt }}(p(\mathbf{H}), \gamma) \leq \overline{\mathrm{C}}_{\text {opt }}(p(\mathbf{H}), \gamma) \\
& =\frac{1}{N} \mathbb{E}\left[\log _{2} \operatorname{det}\left(\mathbf{I}_{N}+\gamma\left(\mathbf{G ~ G}^{H}\right) \circ\left(\boldsymbol{\Omega}^{H}\right)\right)\right] \\
& =\frac{1}{N} \mathbb{E}\left[\log _{2}(1+\gamma \operatorname{tr}(\breve{\mathbf{G}} \circ \breve{\Omega}))\right],
\end{aligned}
$$

where we have made use of property $\operatorname{det}(\mathbf{I}+\gamma \mathbf{A})=1+\gamma \operatorname{tr} \mathbf{A}+\mathcal{O}\left(\gamma^{2}\right)$; also we have ignored the terms with higher order of $\gamma$ for $\gamma \rightarrow 0$; $\breve{\mathbf{~}}=\mathbf{G G}^{H}$; $\operatorname{tr}(\breve{\mathbf{G}} \circ \breve{\mathbf{\Omega}}) ; \operatorname{tr}(\breve{\mathbf{G}} \circ \check{\mathbf{\Omega}})$ denotes the trace of the Hadamard product of the composite channel matrix $(\breve{\mathbf{G}} \circ \breve{\mathbf{\Omega}})$ and

$$
\begin{aligned}
& \frac{1}{N} \mathcal{V}_{(\breve{\mathbf{G}} \odot \mathbf{\Omega})}(\gamma)=\frac{1}{N} \mathbb{E}\left[\log _{2}(1+\gamma \operatorname{tr}(\breve{\mathbf{G}} \circ \breve{\boldsymbol{\Omega}}))\right]
\end{aligned}
$$

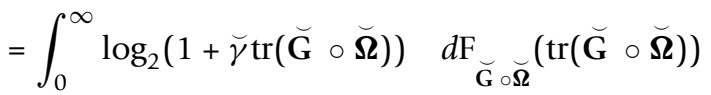

is the Shannon transform of a random square Hadamard composite matrix $(\breve{\mathbf{G}} \circ \check{\mathbf{\Omega}})$ and distributed according to the cumulative distribution function (CDF)

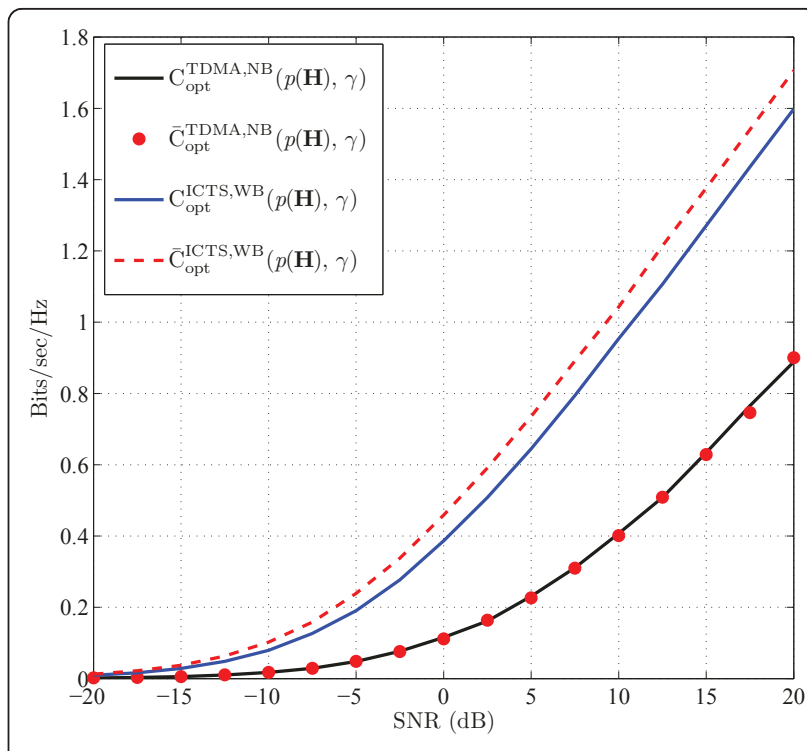

(a) $K=5$

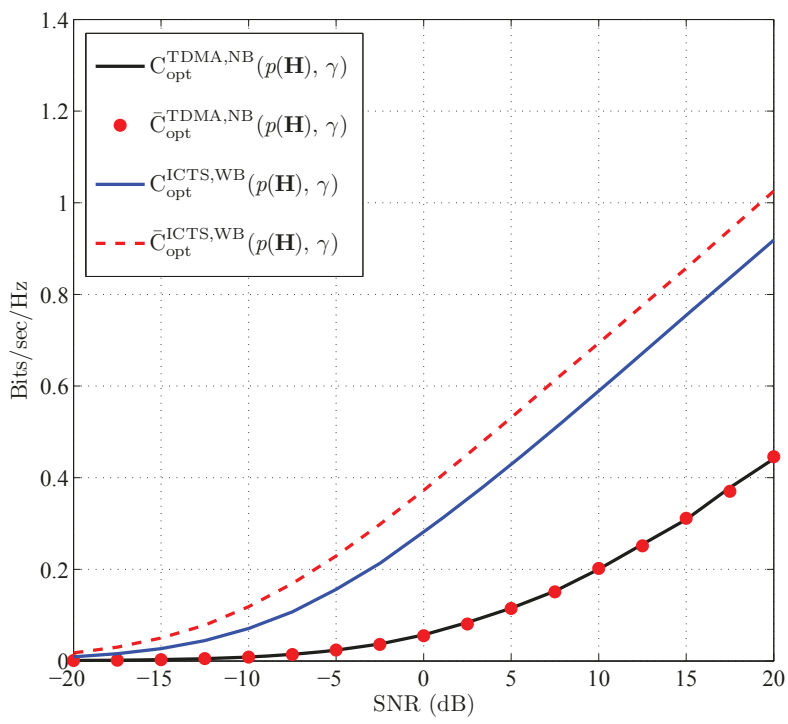

(b) $K=10$

Figure 3 Summary of optimum joint decoding capacity and theoretical Hadamard upper bound on the optimum capacity for the multi-user case when TDMA, NB and ICTS, WB schemes are employed. (a) $K=5$; (b) $K=10$.

denoted by $\mathrm{F}_{\breve{\mathbf{G}} \circ \breve{\mathbf{\Omega}}}(\operatorname{tr}(\breve{\mathbf{G}} \circ \breve{\mathbf{\Omega}}))[17]$, where $\breve{\gamma}=\gamma N^{2}$ and $\gamma=P / \sigma_{z}^{2}$ is the MT transmit power over receiver noise ratio.

Using trace inequality [34], we have an upper bound on (34) as 
Table 1 Summary of theoretical Hadamard upper bound (HUB)

\begin{tabular}{|c|c|c|}
\hline User(s) $(K)$ & Constraints for $\mathrm{C}_{\mathrm{opt}}(p(\mathrm{H}) ; \gamma)=\overline{\mathrm{C}}_{\mathrm{opt}}(p(\mathrm{H}) ; \gamma)$ & Constraints for $\mathrm{C}_{\mathrm{opt}}(p(\mathrm{H}) ; \gamma)<\overline{\mathrm{C}}_{\mathrm{opt}}(p(\mathrm{H}) ; \gamma)$ \\
\hline $\begin{array}{c}K=1 \text { (Cooperative } \\
\text { BS scenario) }\end{array}$ & $\begin{array}{l}\text { i. } \Omega \rightarrow 0 \text {, i.e., low level of inter-cell interference to } \\
\text { the BS of interest. } \\
\text { ii. } \gamma \rightarrow 0 \text {, i.e., the gain inserted by } \operatorname{HUB} \Delta \rightarrow 0 \text { and } \\
\text { is given by } \Delta_{0}=\lim _{\gamma \rightarrow 0} \gamma \mathbb{E}[\operatorname{tr}(\Gamma(P, Q))] \text {. }\end{array}$ & $\begin{array}{l}\Omega \sim \mathcal{U}(0,1) \text { (variable path gain among the MTs and the Bs of interest } \\
\text { due to Uniformly distributed MTs across the cells). }\end{array}$ \\
\hline $\begin{array}{c}\text { K> } 1 \text { (Non- } \\
\text { cooperative BS } \\
\text { scenario) }\end{array}$ & $\begin{array}{l}\text { By employing intra-cell TDMA, intercell Narrowband } \\
\text { (TDMA, NB) scheme. }\end{array}$ & By employing Inter-cell Time Sharing, Wideband (ICTS, WB) scheme. \\
\hline
\end{tabular}

$$
\begin{aligned}
& \mathrm{C}_{\text {opt }}(p(\mathbf{H}), \gamma) \leq \tilde{\mathrm{C}}_{\text {opt }}(p(\mathbf{H}), \gamma) \\
& =\frac{1}{N} \mathbb{E}\left[\log _{2}(1+\gamma \operatorname{tr}(\breve{\mathbf{G}}) \operatorname{tr}(\breve{\Omega}))\right] .
\end{aligned}
$$

If $u=x y$; where $x=\operatorname{tr}(\breve{\mathbf{G}})$ and $y=\operatorname{tr}(\breve{\mathbf{\Omega}})$ then $(36$ can also be expressed as

$$
\begin{aligned}
& \tilde{\mathrm{C}}_{\text {opt }}(p(\mathbf{H}), \gamma)=\int_{0}^{\infty} \log _{2}(1+\widetilde{\gamma} u) d \mathrm{~F}_{\breve{\mathbf{G}} \circlearrowleft \mathbf{\Omega}}(u) \\
& =\int_{0}^{\infty} \log _{2}(1+\breve{\gamma} u) f_{\breve{\mathbf{G}} \circlearrowleft \mathbf{\Omega}}(u) d u .
\end{aligned}
$$

where $f_{\breve{G} \circ \breve{\Omega}}(u)$ is the joint PDF of the $\operatorname{tr}(\check{\mathbf{G}})$ and $\operatorname{tr}(\breve{\Omega})$ which is evaluated as follows in the next subsection.

A. Approximation of PDF of $\operatorname{tr}(\breve{\mathbf{G}} \circ \breve{\mathbf{\Omega}})$

Let $u=x y$ and $v=x$, then the Jacobian is given as

$$
\begin{aligned}
& J\left(\frac{u, v}{x, y}\right)=\left|\begin{array}{ll}
y & x \\
1 & 0
\end{array}\right|=-x=-\frac{u}{y} . \\
& f_{\breve{\mathbf{G}} \odot \mathbf{\Omega}}(u, v) d u d v=f_{\breve{\mathbf{G}} \circlearrowleft \mathbf{\Omega}}(x, y) d x d y=f_{\breve{\mathbf{G}} \circlearrowleft \overline{\boldsymbol{\Omega}}}(x, y) \frac{y}{u} d u d v,
\end{aligned}
$$

so,

$$
f_{\breve{\mathbf{G}} \odot \mathbf{\Omega}}(u, v)=\frac{y}{u} f_{\breve{\mathbf{G}} \odot \boldsymbol{\Omega}}(x, y) .
$$

where we approximate the PDF of $f_{\breve{\mathbf{G}} \circ \widetilde{\boldsymbol{\Omega}}}(x, y)$ of Hadamard product of two random variables $x$ and $y$ as a product of Gaussian and Uniform distributions, respectively, such that their joint PDF can be expressed as

$$
f_{\breve{\mathbf{G}} \circlearrowleft \breve{\Omega}}(x, y)=\frac{1}{\sqrt{2 \pi}} \exp \left(-\frac{x^{2}}{2}\right) f(y),
$$

where $f(y)$ denotes the uniform distribution of MTs. Using (43) and (44), the PDF of the trace of Hadamard product of two composite matrices $\breve{\mathbf{G}}$ and $\breve{\boldsymbol{\Omega}}$ may be approximated as

$$
f_{\breve{\mathbf{G}} \subseteq \mathbf{\Omega}}(u)=\frac{1}{\sqrt{2 \pi}} \int_{0}^{1} \frac{y}{u} \exp \left(-\frac{u^{2}}{2 y^{2}}\right) d y,
$$

by substituting (45) into (40), the analytical HUB on optimum joint decoding capacity can be calculated as

$$
\begin{aligned}
& \tilde{\mathrm{C}}_{\text {opt }}(p(\mathbf{H}), \gamma)=\frac{1}{\sqrt{2 \pi}} \int_{0}^{\infty} \int_{0}^{1} \frac{\gamma}{u} \log _{2}(1+\breve{\gamma} u) \exp \left(-\frac{u^{2}}{2 \gamma^{2}}\right) d y d u, \\
& \tilde{\mathrm{C}}_{\text {opt }}(p(\mathrm{H}), \gamma)=\Theta\left(\breve{\gamma}^{2} G_{4,6}^{5,3}\left(\frac{1}{16 \sigma^{4}} \mid \begin{array}{c}
0, \frac{1}{4}, \frac{3}{4}, 1 \\
0,0,0, \frac{1}{4}, \frac{3}{4}, \frac{1}{2}
\end{array}\right)+\breve{\gamma}^{2} G_{4,6}^{5,3}\left(\frac{1}{16 \breve{\gamma}^{4}} \mid \begin{array}{c}
\frac{1}{4}, \frac{1}{4}, \frac{3}{4}, 1 \\
0, \frac{1}{4}, \frac{1}{2}, \frac{1}{2}, \frac{3}{4}, 0
\end{array}\right)\right. \\
& \left.-4 \sqrt{\pi} G_{3,4}^{4,2}\left(\frac{1}{2 \breve{\gamma}^{2}} \mid \begin{array}{c}
-1,-\frac{1}{2}, 1 \\
-1,-1,-\frac{1}{2}, 0
\end{array}\right)\right)
\end{aligned}
$$

where we have made a use of Meijer's G-Function [35], available in standard scientific software packages, such as Mathematica, in order to transform the integral expression to the closed form and $\Theta=1 / 64 \sqrt{2} \pi^{2} \gamma^{2}$.

\section{Numerical examples and discussions}

In this section, we present Monte Carlo simulation results in order to validate the accuracy of the analytical analysis based on approximation approach for upper bound on optimum joint decoding capacity of $\mathrm{C}$ GCMAC with Uniformly distributed MTs. In the context of Monte Carlo finite system simulations, the MTs gains toward the BS of interest are randomly generated according to the considered distribution and the capacity is calculated by the evaluation of capacity formula (14). Using (34), the upper bound on the optimum capacity is calculated. It can be seen from Figure 4 that the theoretical upper bound converges to the actual capacity under constraints like low SNRs (compare the black solid curve with the red dashed curve). In the context of mathematical analysis which is the main contribution of this article, (47) is utilized to compare the analytical upper bound based on proposed analytical approach with the theoretical upper bound based on simulations. It can also be seen from Figure 4 that the proposed approximation shows comparable results over the entire range of SNR (compare the blue dotted curve and the red dashed curve). However, it is to note that an 


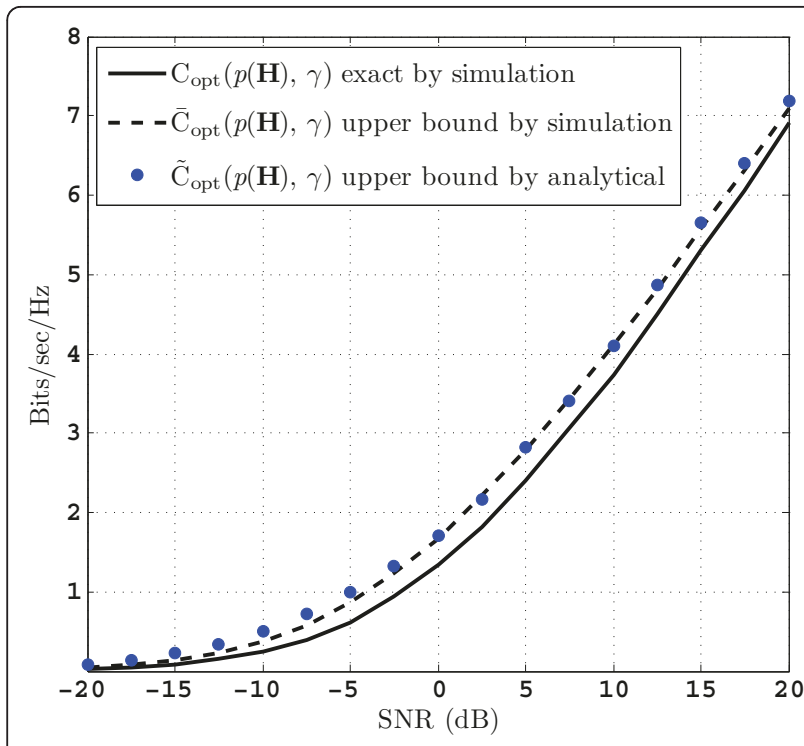

Figure 4 Summary of Hadamard upper bound on optimum joint decoding capacity of C-GMAC for variable user-density across the cells; the black solid curve illustrates actual capacity using (14) obtained by Monte Carlo simulations; the red dashed and dotted curves illustrate HUB obtained by Monte Carlo simulations and analytical analysis using (34) and (47), respectively. The simulation curves are obtained after averaging 10,000 Monte Carlo trials of the composite channel $\mathbf{H}$ with $N=6$ and $K=1$.

analytical HUB on optimum joint decoding capacity of multi-cell setup is comparably tighter in the high SNR regime as compared to the HUB in the low SNR regime. The proposed approximation based approach is useful to represent the capacity for the realistic multi-cell setup, i.e., variable user-density and therefore variable channel path gain toward the BS of interest.

A figure of merit utilized in cellular communication, which is referred to as mean ASE

$$
A_{e}=\mathrm{C}_{\mathrm{opt}} / \pi R^{2} \quad \mathrm{bits} / \mathrm{s} / \mathrm{Hz} / \mathrm{km}^{2}
$$

averaged over a large number of fading realizations $g_{B_{j} T_{j+i}}^{l}$ and channel path gain $\Omega_{B_{j} T_{j+i}}^{l}$ for all $(j, i)=(1 \ldots N)$ $\times(0, \pm 1)$ and $K$ users [36]. Further, we assumed that the range of cell radius $R$ is $0.1-1 \mathrm{Km}$ for the system under consideration. The ASE quantifies the sum of maximum bit rates/Hz/unit area supported by the BS in a cell [36]. Figure 5a,b shows the ASE calculated for $\gamma=$ $-10 \mathrm{~dB}$ and $\gamma=15 \mathrm{~dB}$, respectively. It can be seen that the analytical HUB on optimum joint decoding capacity based on proposed approximation approach is close to the Monte Carlo simulation results within the entire cell radii for high SNR. On the other side for low SNRs, the HUB is loose up to 500 meters approximately of cell radius and comparably tighter within the higher range of cell radii.

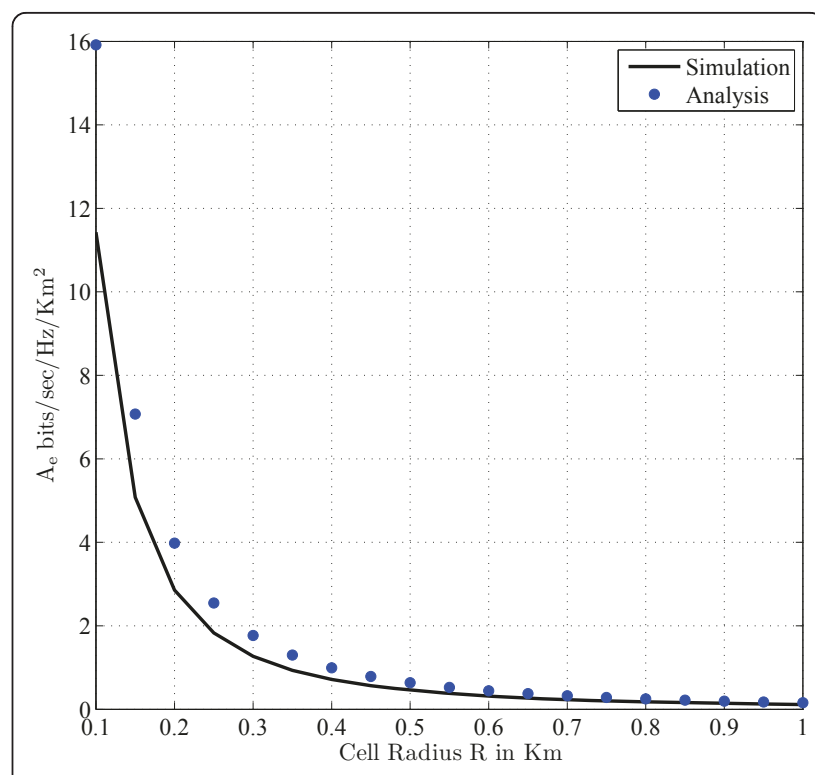

(a) $\gamma=-10 \mathrm{~dB}$

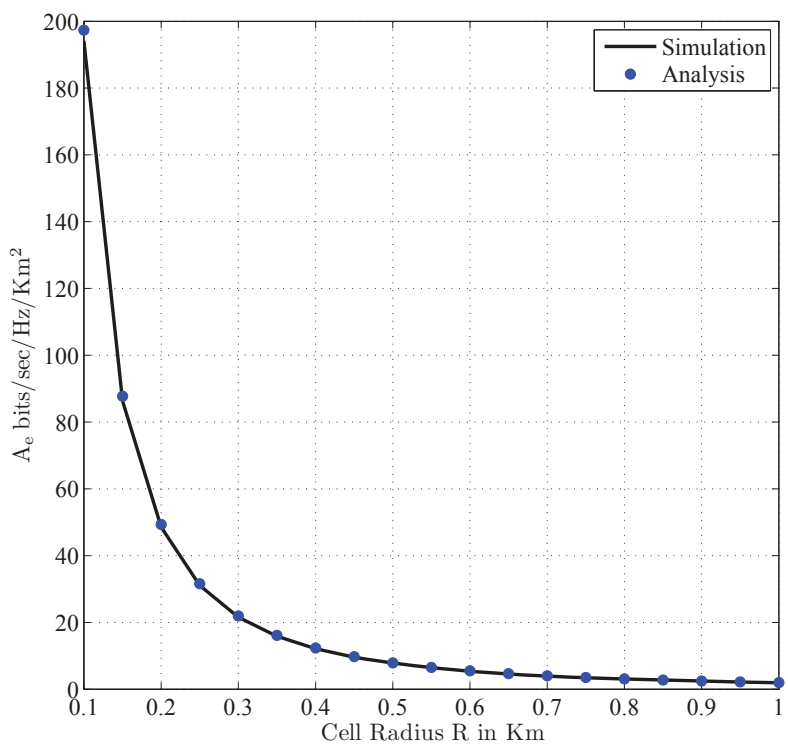

(b) $\gamma=15 \mathrm{~dB}$

Figure 5 Area mean spectral efficiency (bits $/ \mathrm{s} / \mathrm{Hz} / \mathrm{km}^{2}$ ) versus the cell radius: (a) $\gamma=-10 \mathrm{~dB}$; (b) $\gamma=15 \mathrm{~dB}$.

\section{Conclusion}

The analytical upper bound referred to as HUB is derived on optimum joint decoding capacity for Wyner C-GCMAC under realistic assumptions: uniformly distributed MTs across the adjacent cells; and the finite number of cooperating BSs arranged in a circular configuration. New analytical approach have been reported to derive an information theoretic upper bound on the 
optimum joint decoding capacity of circular Wyner GCMAC. This approach is based on the approximation of the PDF of trace of composite Hadamard product matrix $(\mathbf{G} \circ \mathbf{\Omega})$ by employing the Hadamard inequality. A closed form expression has been derived to capture the effect of variable user-density in GCMAC. The proposed analytical approach has been validated by using Monte Carlo simulations for variable user-density cellular system. It has been shown that a reasonably tighter upper bound on optimum joint decoding capacity can be obtained by exploiting Hadamard inequality for realistic scenarios in cellular network. The importance of the methodology presented here lies in the fact that it allows a realistic representation of the MT's spatial arrangement. Therefore, this approach can be further exploited in order to investigate the various practical MT distributions and their effect on the optimum joint decoding capacity of system under consideration.

\section{Appendix A}

\section{An Alternate Proof Of (18)}

Proof: We derive an alternate version of (17) for rank one matrices $\mathbf{G}$ and $\boldsymbol{\Omega}$ which also proves the Hadamard inequality (18). Let us define $\mathbf{G}=\boldsymbol{u} \boldsymbol{v}^{H}$ and $\boldsymbol{\Omega}=\boldsymbol{w} \boldsymbol{z}^{H}$; where $\boldsymbol{u}, \boldsymbol{v}, \boldsymbol{w}, \boldsymbol{z}$ are $N \times 1$ column vectors which corresponds to a vector channel between a user in any of $j$ th cell and $N$ BSs. Then,

$$
\mathbf{G} \circ \boldsymbol{\Omega}=(\boldsymbol{u} \circ \boldsymbol{w})(\boldsymbol{v} \circ \boldsymbol{z})^{H},
$$

also is of rank at most one, and we calculate that

$$
\begin{aligned}
& (\mathbf{G} \circ \boldsymbol{\Omega})(\mathbf{G} \circ \boldsymbol{\Omega})^{H}=(\boldsymbol{u} \circ \boldsymbol{w})(\boldsymbol{v} \circ \boldsymbol{z})^{H}(\boldsymbol{v} \circ \boldsymbol{z})(\boldsymbol{u} \circ \boldsymbol{w})(\mathrm{A} .50) \\
& =\|\boldsymbol{v} \circ \boldsymbol{z}\|^{2}(\boldsymbol{u} \circ \boldsymbol{w})(\boldsymbol{u} \circ \boldsymbol{w})^{H},
\end{aligned}
$$

also we have

$$
\begin{aligned}
& \mathrm{GG}^{H}=\left(\boldsymbol{u} v^{H}\right)\left(u v^{H}\right)=\|\boldsymbol{v}\|^{2}\left(u u^{H}\right), \\
& \boldsymbol{\Omega} \boldsymbol{\Omega}^{H}=\left(\boldsymbol{w} z^{H}\right)\left(\boldsymbol{w} z^{H}\right)=\|z\|^{2}\left(\boldsymbol{w} \boldsymbol{w}^{H}\right),
\end{aligned}
$$

this gives the formula

$$
\begin{aligned}
& \left(\mathrm{G} \mathrm{G}^{H}\right) \circ\left(\boldsymbol{\Omega} \boldsymbol{\Omega}^{H}\right)=\|\boldsymbol{v}\|^{2}\|z\|^{2}\left(\boldsymbol{u} u^{H}\right) \circ\left(\boldsymbol{w} \boldsymbol{w}^{H}\right), \\
& =\|\boldsymbol{v}\|^{2}\|z\|^{2}(u \boldsymbol{w}) \circ\left(\boldsymbol{u}^{H} \boldsymbol{w}^{H}\right),
\end{aligned}
$$

comparing the formulas (A.51) and (A.55), we obtain the identity

$$
\left(\mathbf{G ~ G}^{H}\right) \circ\left(\boldsymbol{\Omega} \boldsymbol{\Omega}^{H}\right)=\frac{\|\boldsymbol{v}\|^{2}\|z\|^{2}}{\|\boldsymbol{v} \circ \boldsymbol{z}\|^{2}}(\mathbf{G} \circ \boldsymbol{\Omega})(\mathbf{G} \circ \boldsymbol{\Omega})^{H} .
$$

In particular, since the norm is sub-multiplicative relative to the Hadamard product

$$
\|\boldsymbol{v} \circ \boldsymbol{z}\| \geq\|\boldsymbol{v}\|\|\boldsymbol{z}\|,
$$

finally, we can prove that

$$
\mathbf{G G}^{H} \circ \boldsymbol{\Omega} \boldsymbol{\Omega}^{H} \geq(\mathbf{G} \circ \boldsymbol{\Omega})(\mathbf{G} \circ \boldsymbol{\Omega})^{H} .
$$

This completes the proof.

Remarks: The result (A.56) can be applied to the correlated scenario where the rank of the fading channel matrix may reduce to $1[2,15,26]$. Alternatively, the proof can be extended for channel matrix of any rank $L$. As an example, if $\mathbf{A}$ is a diagonalizable matrix of size $N$ $\times N$ with rank $L$. Then, there are $L$ square rank one matrices given as $\mathbf{A}_{1}, \mathbf{A}_{2}, \ldots, \mathbf{A}_{L}$, such that $\mathbf{A}=\mathbf{A}_{1}+\mathbf{A}_{2}$ $+\ldots+\mathbf{A}_{L}$ and an alternative proof can be derived for such matrices.

\section{ENDNOTES}

${ }^{a}$ MTs are also referred to as users and is interchangeably used throughout the article.

${ }^{b} T_{j+1} \triangleq T_{j+1} \bmod N$.

${ }^{c}$ Throughout this article, $\mathbf{H}_{N, K}, \mathbf{G}_{N, K}$ and $\Omega_{N, K}$ refers to the channel matrices corresponding to $N$ number of cells and $K$ users per cell in a C-GCMAC. For brevity, the channel matrices will be expressed as $\mathbf{H}, \mathbf{G}$ and $\Omega$, respectively, unless it is necessary to emphasis the number of cells and the number of users.

${ }^{\mathrm{d}}$ Here, we used Matlab format to express row vector. For an example, $\boldsymbol{\Omega}(1$, :) shows First row vector of matrix $\Omega$.

${ }^{\mathrm{e}}$ As an example, for $N=6$ and $K=1$, the partial permutation matrices are $\mathbf{P} \in \mathbb{P}^{36 \times 6}$ and $\mathbf{Q} \in \mathbb{P}^{36 \times 30}$ [26].

${ }^{\mathrm{f}}$ Terms with higher order of $\gamma$ are ignored $\Leftrightarrow \gamma^{x} \approx 0$; $\forall x=2,3, \ldots$ [33].

\section{Acknowledgements}

The authors would like to acknowledge the financial support of Picsel Technologies Ltd, Glasgow, UK and KAUST, KSA. This study was presented in part at IEEE International Symposium Wireless Communications and Systems, ISWCS'2010, York, UK, September 2010.

\section{Author details}

${ }^{1}$ Division of Physical Sciences and Engineering, King Abdullah University of Science and Technology, KAUST, Thuwa1 23599-6900, Makkah Province, Kingdom of Saudi Arabia ${ }^{2}$ Department of Electronic and Electrical Engineering, University of Strathclyde, 204 George Street, Glasgow Gl 1XW UK

\section{Competing interests}

The authors declare that they have no competing interests.

Received: 5 November 2010 Accepted: 25 September 2011 Published: 25 September 2011

\section{References}

1. A Ghosh, J Zhang, JG Andrews, R Muhamed, Fundamentals of LTE, Boston, Massachusetts, MA, USA: Prentice-Hall, Sep. 2010 
2. G Kramer, I Maric, RD Yates, Cooperative Communications (Foundations and Trends in Networking), Hanover, Massachusets, MA, USA: now Publishers, Inc, Jun. 2007

3. GJ Foschini, MJ Gans, On the limits of wireless communications in a fading environment when using multiple antennas. Springer Jour Wireless Personal Communs. 6(3), 311-335 (Mar. 1998)

4. O Somekh, BM Zaidel, Sum rate characterization of joint multiple cell-site processing. IEEE Trans Inform Theory 53(12), 4473-4497 (Dece. 2007)

5. S Shamai, AD Wyner, Information-theoretic considerations for symmetric cellular multiple-access fading channels - Part I. IEEE Trans Inform Theory 43(6), 1877-1894 (Nov. 1997). doi:10.1109/18.641553

6. S Shamai, AD Wyner, Information-theoretic considerations for symmetric cellular multiple-access fading channels - Part II. IEEE Trans Inform Theory 43(6), 1895-1911 (Nov. 1997). doi:10.1109/18.641554

7. AD Wyner, Shannon-theoretic approach to a Gaussian cellular multipleaccess channel. IEEE Trans Inform Theory 40(6), 1713-1727 (Nov. 1994). doi:10.1109/18.340450

8. IE Teletar, Capacity of multi-antenna Gaussian channels. European Trans Telecomm. 10, 585-595 (Nov. 1999). doi:10.1002/ett.4460100604

9. JG Andrews, W Choi, RW Heath, Overcoming interference in spatial multiplexing MIMO wireless networks. IEEE Mag Wireless Communs. 14(6), 95-104 (Dec. 2007)

10. G Boudreau, J Panicker, N Guo, R Chang, N Wang, S Vrzic, Interference coordination and cancellation for $4 \mathrm{G}$ networks. IEEE Mag Communs. 47(4), 77-81 (Apr. 2009)

11. J Zhang, R Chen, JG Andrews, A Ghosh, RW Heath Jr, Networked MIMO with clustered linear precoding. IEEE Trans Wireless Communs. 8(4), 1910-1921 (Apr. 2009)

12. MZ Shakir, TS Durrani, M-S Alouini, On the optimum joint decoding capacity of Wyner Circular GCMAC by exploiting Hadamad inequality. in Proc IEEE Intl Symp Wireless Communs and Systems, ISWCS' 2010, York, United Kingdom, 785-789 (Sep. 2010)

13. O Somekh, O Simeone, HV Poor, S Shamai (Shitz), Throughput of cellular uplink with dynamic user activity and cooperative base-stations. Proc IEEE Inform Theory Workshop, ITW' 2009, Taormina, Italy, 610-614 (Oct. 2009)

14. S Shamai (Shitz), O Somekh, BM Zaidel, Multi-cell communications: an information theoretic perspective. in Proc Joint Workshop Commun and Coding, JWCC'04, Donnini, Florence, Italy (Oct. 2004)

15. O Somekh, O Simeone, Y Bar-Ness, AM Haimovich, U Spagnolini, S Shamai (Shitz), Distributed Antenna Systems: Open Architecture for Future Wireles Communications, Chapter: An Information Theoretic View of Distributed Antenna Processing in Cellular Systems, New York, NY, USA: Auerbach Publications, CRC Press (May 2007)

16. P Marsch, S Khattak, G Fettweis, A framework for determining realistic capacity bounds for distributed antenna systems. Proc IEEE Inform Theory Workshop, ITW'2006, Punta del Este, Uruguay, 571-575 (Oct. 2006)

17. AM Tulino, S Verdu, Random Matrix Theory and Wireless Communications (Foundations and Trends in Commus. and Inform. Theory), Hanover, Massachusets, MA, USA: now Publisher Inc, (Jun. 2004)

18. TM Cover, JA Thomas, Elements of Information Theory, 2nd edn. (New Jersey, USA: John Wiley and Sons, Inc, 2006)

19. O Simeone, O Somekh, HV Poor, S Shamai (Shitz), Local base station cooperation via finite capacity links for the uplink of linear cellular networks. IEEE Trans Inform Theory 55(1), 190-204 (Jan. 2009)

20. D Aktas, MN Bacha, JS Evans, SV Hanly, Scaling results on the sum capacity of cellular networks with MIMO links. IEEE Trans Inform Theory. 52(7), 3264-3274 (Jul. 2006)

21. D Kaltakis, MA Imran, C Tzaras, Information theoretic capacity of cellular multiple access channel with shadow fading. IEEE Trans Communs. 58(5), 1468-1476 (May 2010)

22. J Xu, J Zhang, JG Andrews, On the accuracy of the Wyner model in cellular networks. IEEE Trans Wireless Communs. 10(9), 3098-3109 (Sep. 2011)

23. S Chatzinotas, MA Imran, C Tzaras, On the capacity of variable density cellular Systems under multi-cell decoding. IEEE Let Communs. 12, 496-498 (Jul. 2008)

24. E Katranaras, MA Imran, C Tzaras, Uplink capacity of a variable density cellular system with multi-cell processing. IEEE Trans Communs. 57(7), 2098-2108 (Jul. 2009)

25. Y Liang, A Goldsmith, Symmetric rate capacity of cellular systems with cooperative base stations. in Proc IEEE Conf Global Communs., GLOBECOM'2006, San Francisco, California, CA, USA, 1-5 (Nov. 2006)
26. MZ Shakir, Cellular MIMO networks-channel modeling, decomposition and information theoretic perspectives, PhD thesis, University of Strathclyde, Glasgow, UK, (Mar. 2010)

27. RM Gray, Toeplitz and circulant matrices: a review, http://ee.stanford.edu/ gray/toeplitz.pdf (2002)

28. R Goodman, Finite fourier transforms circulant matrices and fast fourier transform, http://www.math.rutgers.edu/courses/550A/550-f07/fftnotes.pdf (2007)

29. T Amemiya, Advanced Econometrics, (Cambridge, Massachusetts, MA, USA: Harvard University Press, 1985)

30. RA Horn, CR Johnson, Matrix Analysis, (Cambridge, United Kingdom: Cambridge University Press, 1985)

31. A Kilicman, Z Al-Zhour, On the connection between Kronecker and Hadamard convolution products of matrices and some applications. Hindawi Jour Inequalities and Applications 10 (Apr. 2009). Article ID: 736243

32. G Visick, A quantitative version of the observation that the Hadamard product is a principal sub-matrix of the Kronecker product. Elsevier Jour Linear Algebra and its Applications 304, 45-68 (Jan. 2000). doi:10.1016/ S0024-3795(99)00187-1

33. RA Beezer, A First course in linear algebra, version 2.20 (2009)

34. Z Ulukok, R Turkmen, On some matrix trace inequalities. Hindawi Jour Inequalities and Applications 1-8 (2010). Article 1D: 201486

35. M Abramowitz, IA Stegun, Handbook of Mathematical Functions with Formulas, Graphs, and Mathematical Tables, 9th edn. (New York, USA: Dover Publication, Inc, 1972)

36. M-S Alouini, A Goldsmith, Area spectral efficiency of cellular mobile radio systems. IEEE Trans Vehicular Technology 48(4), 1047-1066 (Jul. 1999). doi:10.1109/25.775355

doi:10.1186/1687-1499-2011-110

Cite this article as: Shakir et al:: Hadamard upper bound on optimum joint decoding capacity of Wyner Gaussian cellular MAC. EURASIP Journal on Wireless Communications and Networking 2011 2011:110.

\section{Submit your manuscript to a SpringerOpen ${ }^{\mathcal{O}}$ journal and benefit from:}

- Convenient online submission

- Rigorous peer review

- Immediate publication on acceptance

- Open access: articles freely available online

- High visibility within the field

- Retaining the copyright to your article

Submit your next manuscript at $>$ springeropen.com 